\title{
Overexpression of CmMYB15 provides chrysanthemum resistance to aphids by regulating the biosynthesis of lignin
}

\author{
Cong $A n^{1}$, Liping Sheng ${ }^{1}$, Xinping Du' ${ }^{1}$ Yinjie Wang ${ }^{1}, Y_{i}$ Zhang ${ }^{1}$, Aiping Song ${ }^{1}$, Jiafu Jiang ${ }^{1}$, Zhiyong Guan' ,
} Weimin Fang ${ }^{1}$, Fadi Chen ${ }^{1}$ and Sumei Chen ${ }^{1}$

\begin{abstract}
MYB transcription factors are widely involved in the development of and physiological processes in plants. Here, we isolated the chrysanthemum R2R3-MYB family transcription factor CmMYB15, a homologous gene of AtMYB15. It was demonstrated that CMMYB15 expression was induced by aphids and that CmMYB15 could bind to AC elements, which usually exist in the promoter of lignin biosynthesis genes. Overexpression of CmMYB15 in chrysanthemum enhanced the resistance of aphids. Additionally, the content of lignin and the expression of several lignin biosynthesis genes increased. In summary, the results indicate that CMMYB15 regulates lignin biosynthesis genes that enhance the resistance of chrysanthemum to aphids.
\end{abstract}

\section{Introduction}

Members of the MYB family, one of the largest transcription factor families in plants, have a common conserved N-terminal MYB domain consisting of imperfect amino acid repeats of $\sim 52$ amino acids ${ }^{1}$. These members are generally divided into four classes, 1R-, R2R3-, 3Rand 4R-MYB proteins, according to the number of adjacent repeats, with the R2R3-MYB subfamily comprising one of the largest families of transcription factors in plants $^{2}$. MYBs have been shown to be key factors involved in vital regulatory networks that regulate physiological and biochemical processes, for instance, plant development, responses to biotic and abiotic stress ${ }^{3}$, and primary and secondary metabolism, such as flavonoid biosynthesis and phenylpropanoid metabolism ${ }^{4,5}$.

The lignin biosynthetic pathway is among the important branches of the phenylpropanoid metabolic pathway, as lignin is the second most abundant plant biopolymer.

\footnotetext{
Correspondence: Sumei Chen (chensm@njau.edu.cn)

${ }^{1}$ State Key Laboratory of Crop Genetics and Germplasm Enhancement, the Key Laboratory of Landscaping, Ministry of Agriculture, College of Horticulture, Nanjing Agricultural University, Nanjing 210095, China

These authors contributed equally: Cong An, Liping Sheng
}

Lignin is a major secondary cell wall component that provides mechanical support for plants and is also a chemical and physical barrier that provides defense against biotic and abiotic stress ${ }^{6}$. The precursors of lignin, which are phenolic compounds, can participate in plantpathogen interaction pathways, such as that of the interaction between grapevine and Erysiphe necator ${ }^{7}$. A relatively high accumulation of lignin increases the strength of birch leaves, which reduces vulnerability to the autumnal moth, Epirrita autumnata ${ }^{8}$. Lignin enhances resistance to cell wall-degrading enzymes (CWDEs), which are produced by fungal necrotrophs, therefore preventing the diffusion of pathogen-produced toxins ${ }^{9}$. Enzymes participating in lignin biosynthesis include phenylalanine ammonia lyase (PAL), 4hydroxycinnamoyl-CoA ligase (4CL), caffeoyl shikimate esterase (CSE), p-coumarate 3-hydroxylase (C3H), caffeoyl-CoA O-methyltransferase (CCoAOMT), cinnamoyl-CoA reductase (CCR), ferulate 5-hydroxylase (F5H), caffeic acid/5-hydroxyferulic acid Omethyltransferase (COMT), and cinnamyl alcohol dehydrogenase $(C A D)^{10,11}$. Analysis of promoter sequences showed that most of these genes contain one or more AC-

\section{(c) The Author(s) 2019}

(c) (i) Open Access This article is licensed under a Creative Commons Attribution 4.0 International License, which permits use, sharing, adaptation, distribution and reproduction cc) in any medium or format, as long as you give appropriate credit to the original author(s) and the source, provide a link to the Creative Commons license, and indicate if changes were made. The images or other third party material in this article are included in the article's Creative Commons license, unless indicated otherwise in a credit line to the material. If material is not included in the article's Creative Commons license and your intended use is not permitted by statutory regulation or exceeds the permitted use, you will need to obtain permission directly from the copyright holder. To view a copy of this license, visit http://creativecommons.org/licenses/by/4.0/. 
rich elements in their promoters, except for $\mathrm{F} 5 \mathrm{H}^{12}$. Transcription factors such as MYB proteins regulate the expression of these genes by binding to $\mathrm{AC}$ elements. AtMYB46 activates specific pathways of secondary cell wall biosynthesis and simultaneously upregulates AtMYB85, a downstream secondary cell wall-associated transcription factor ${ }^{13}$. AtMYB58 and AtMYB63 specifically activate lignin biosynthetic genes, which leads to the ectopic deposition of lignin in cells that are normally unlignified $^{6}$. Similarly, PtoMYB216, an MYB-encoding gene in Populus tomentosa, was also found to activate lignin upstream biosynthetic genes ${ }^{14}$. On the other hand, Eriobotrya japonica EjMYB1, a transcriptional activator, and $E j M Y B 2$, a repressor, have an antagonistic relationship in terms of lignification, as the activator and repressor compete with each other for interaction with AC elements ${ }^{15}$. PdMYB221 negatively regulates secondary cell wall formation by binding to the AC elements in the promoter of PdCESA8, PdGT47C, and PdCOMT2, leading to decreased lignin production ${ }^{16}$. Previous studies have indicated that several members of the MYB family regulate the synthesis of lignin. However, if other members of the MYB family are involved in lignin biosynthesis, their regulatory mechanism remains to be elucidated.

Aphids are major agricultural pests that directly cause damage to plants by depriving them of nutrients, and aphids are also vectors for viral pathogens ${ }^{17}$. Chrysanthemum (Chrysanthemum morifolium) is a valuable ornamental species that is vulnerable to Macrosiphoniella sanborni. Aphid infestations hinder not only vegetative growth but also the quality and yield of chrysanthemum flowers. Our previous studies showed that chrysanthemum has evolved a number of strategies to cope with aphid infestation, such as increased defensive enzyme activity $^{18}$ and gene expression reprogramming ${ }^{19,20}$. We previously found that $C m M Y B 19$, a chrysanthemum R2R3-MYB gene, improved the resistance of chrysanthemum to aphids by promoting the biosynthesis of $\operatorname{lignin}^{21}$. In the present study, we isolated chrysanthemum CmMYB15, a gene homologous to AtMYB15, and found that it played a role in lignin biosynthesis and aphid resistance by regulating several downstream genes different from CmMYB19. This study will lay a foundation for the understanding of the physical defense mechanisms of chrysanthemum when challenged by aphids.

\section{Results}

Isolation and sequence analyses of CmMYB15

CmMYB15 was isolated from 'Nannong Xunzhang' chrysanthemum. The CmMYB15 (KT763374) gene consists of $890 \mathrm{bp}$, with a $756 \mathrm{bp}$ open reading frame (ORF) encoding a 251 amino acid polypeptide. CmMYB15 belongs to the R2R3-MYB subfamily, as it has two conserved MYB domains: an R2MYB domain between aa
16-61 and an R3MYB domain between aa 69-112 (Fig. 1a). Phylogenetic analysis indicated that this gene has 32.73\% similarity with AtMYB15 (Fig. 1b).

\section{CmMYB15 is localized to the cell nucleus}

To determine the subcellular localization of CmMYB15, $2 \times 35 \mathrm{~S}::$ CmMYB15-GFP and a negative control, $2 \times 35 \mathrm{~S}$ : GFP, were separately transformed into onion epidermal cells. GFP signals were detected in the nucleus of the cells transformed with $2 \times 35 \mathrm{~S}:: \mathrm{CmMYB} 15-\mathrm{GFP}$, indicating that CmMYB15 was nuclear localized. The GFP signal was detected in both the cytoplasm and nucleus of the cells transformed with $2 \times 35 \mathrm{~S}$ ::GFP (positive control) (Fig. 2).

\section{Transcriptional activation of CmMYB15 and the binding ability of CmMYB15 with AC elements}

Yeast harboring pGBKT7-CmMYB15, or a pCL1 positive control, grew well on $\mathrm{SD} / \mathrm{His}^{-} / \mathrm{Ade}^{-}$medium and turned blue on the SD/His $/ \mathrm{Ade}^{-}$medium with $\mathrm{X}-\alpha$-gal. The negative control transformed with pGBKT7 was unable to grow on the SD/His ${ }^{-} / \mathrm{Ade}^{-}$medium (Fig. 3a), indicating that CmMYB15 has transcriptional activation activity. A yeast one-hybrid assay showed that cells transformed with pHISi-AC-I, pHISi-AC-II or pHISi-AC$\mathrm{III}$ and its corresponding mutated form pHISi-mAC grew well on $\mathrm{SD} / \mathrm{His}^{-} / \mathrm{Ura}^{-}$medium but not on medium with 25 mM 3-amino-1,2,4-triazole (3-AT) (Fig. 3b). Bait Y1H cells (those harboring $\mathrm{AC}$ elements or a mAC element) transformed with pGADT7-CmMYB15 could grow on $\mathrm{SD} / \mathrm{His}^{-} / \mathrm{Ura}^{-} / \mathrm{Leu}^{-}$medium. In contrast to cells expressing pHISi-mAC, cells expressing pHISi-AC-I, pHISi-ACII, or pHISi-AC-III could grow on medium supplemented with $25 \mathrm{mM} 3$-AT (Fig. 3b), suggesting that CmMYB15 could bind all three types of AC elements.

\section{The gene CmMYB15 is highly expressed in leaves and stems and is induced by aphid infestation}

The expression of CmMYB15 was detected in the root, stem and leaf tissues of 'Nannong Xunzhang'. CmMYB15 had the lowest abundance in the roots, while it was highly expressed in the leaves and stems. The expression levels in the stems and leaves were 8.63 times and 15.79 times the level in the roots, respectively (Fig. 4a). In response to aphid infestation, the expression level of CmMYB15 in the aphid-infested plants was 6.84 times greater than that in the control plants at $3 \mathrm{~h}$. During $3 \mathrm{~h}$ to $12 \mathrm{~h}$ after aphid infestation, the expression level of CmMYB15 remained significantly higher than that of the control. The expression level of CmMYB15 was affected by mock puncture except at $-2 \mathrm{~h}, 12$, and $24 \mathrm{~h}$; however, the changes in the expression level of CmMYB15 in response to puncture were much less drastic than those in response to infestation by aphid (Fig. 4b). 
(a)

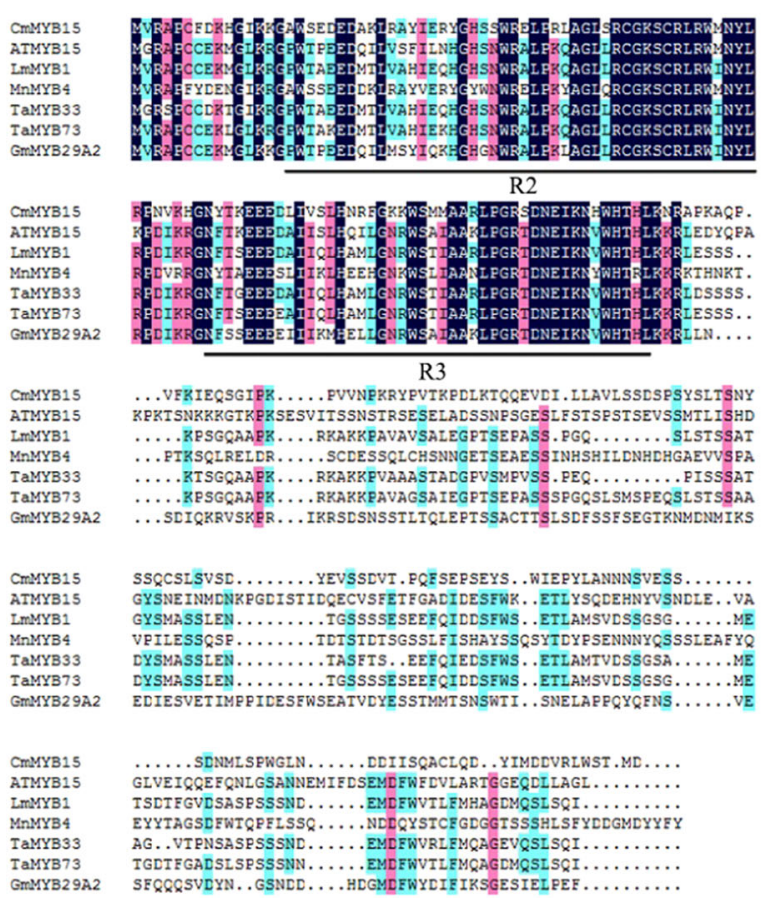

(b)

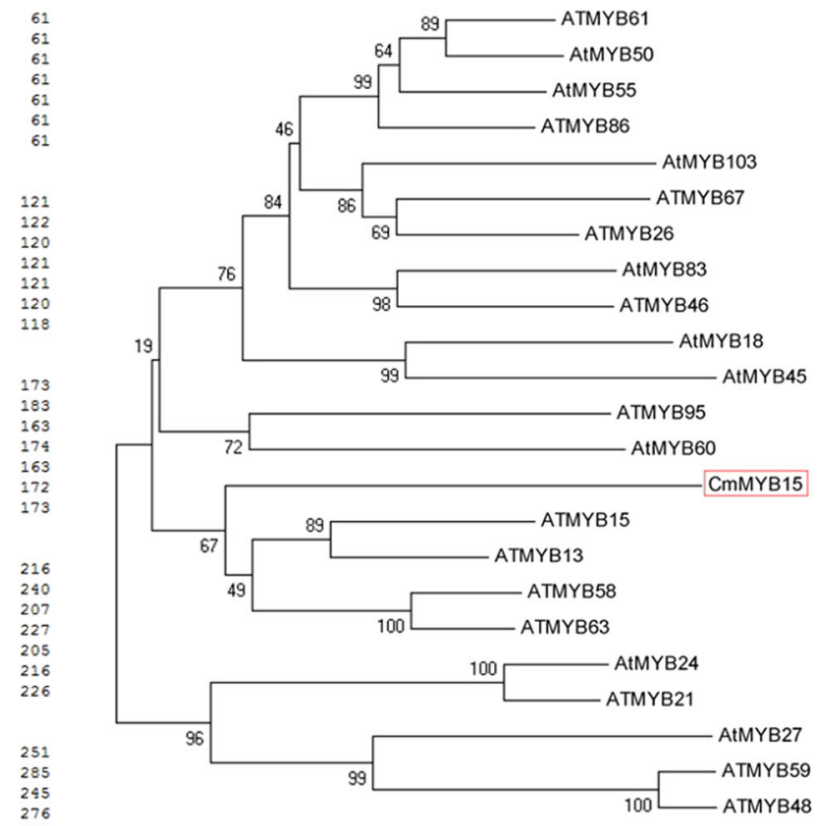

$\longmapsto 0.05$

Fig. 1 Putative peptide sequences of CmMYB15 and other MYB proteins. a Alignment of the putative amino acid sequence of CmMYB15 (boxed) with that of related MYB proteins that feature an R2MYB domain or an R3MYB domain (underlined), b Phylogenetic analysis of the relationships between CmMYB15 and other MYB proteins from Arabidopsis. The sequence details are as follows: AtMYB15 (AT3G23250.1), LmMYB1 (ADN96004.1), MnMYB4 (XP_010095860.1), TaMYB33 (AEO21928.1), TaMYB73 (XP_020169006.1), GmMYB29A2 (BAA81732.1), ATMYB61 (AT1G09540.1), AtMYB50 (AT1G57560.1), AtMYB55 (AT4G01680.1), ATMYB86 (AT5G26660.1), AtMYB103 (AT1G63910.1), ATMYB67 (AT3G12720.1), ATMYB26 (AT3G13890.1), AtMYB83 (AT3G08500.1), ATMYB46 (AT5G12870.1), AtMYB18 (AT4G25560.1), AtMYB45 (AT3G48920.1), ATMYB95 (AT1G74430.1), AtMYB60 (AT1G08810.1), ATMYB13 (AT1G06180.1), ATMYB58 (AT1G16490.1), ATMYB63 (AT1G79180.1), AtMYB24 (AT5G40350.1), ATMYB21 (AT3G27810.1), AtMYB27 (AT3G53200.1), ATMYB59 (AT5G59780.3), and ATMYB48 (AT3G46130.1)

\section{Overexpression of CmMYB15 inhibited the population of aphids on chrysanthemum}

Putative CmMYB15 overexpression lines were identified by PCR using HptII primers (Fig. 5a), and the transcriptional level of $C m M Y B 15$ was measured by qRT-PCR using CmMYB15-specific primers (Fig. 5b). Two independent transgenic lines, CmMYB15-ox3 and CmMYB15-ox4, which presented a higher gene expression level of CmMYB15, were selected for additional aphid resistance assays. The aphids were more widely distributed on the WT plants than on transgenic lines at 21 days after infestation (Fig. 5c). At 11 days after infestation, the number of aphids began to increase rapidly in the control group (WT) plants and reached an average of 270.83 aphids per plant, while the number of aphids on the CmMYB15-ox3 and CmMYB15-ox4 plants were, on average, 153.45 and 236.80 per plant on day 21 (Fig. 5d). CmMYB15-overexpressing plants had a lower average multiplication rate (MR) of 30.69 for CmMYB15-ox3 and 47.36 for CmMYB15-ox4 in comparison to the WT MR of
54.17. Compared to the WT plants, the overexpression lines had higher average inhibition rates (IRs). The IRs for CmMYB15-ox3 and CmMYB15-ox4 in comparison to WT were $43.34 \%$ and $12.57 \%$, respectively (Table 1 ).

\section{Overexpression of $C m M Y B 15$ enhanced the lignin content and expression of lignin biosynthesis genes}

The acetyl bromide procedure ${ }^{23}$ was used to analyze the lignin content of two CmMYB15 overexpression lines. The lignin content of CmMYB15-ox3 and CmMYB15ox4 was $6.56 \%$ and $6.27 \%$ respectively, while the lignin content of the WT was $5.97 \%$ (Fig. 6a).

The expression levels of genes in the lignin biosynthesis pathway, including CmPAL1, Cm4CL1, CmC3H1, CmCSE, CmCCoAMOT1, CmCCR1, CmF5H1, CmCOMT, and $C m C A D 6$, were also quantified. The expression levels of Cm4CL1, CmCCR1, CmF5H1, and CmCAD6 were higher in the overexpression plants than in the WT plants; among these, compared to the other genes analyzed, the expression of $C m C C R 1$ and CmCAD6 increased significantly (Fig. 6b). 

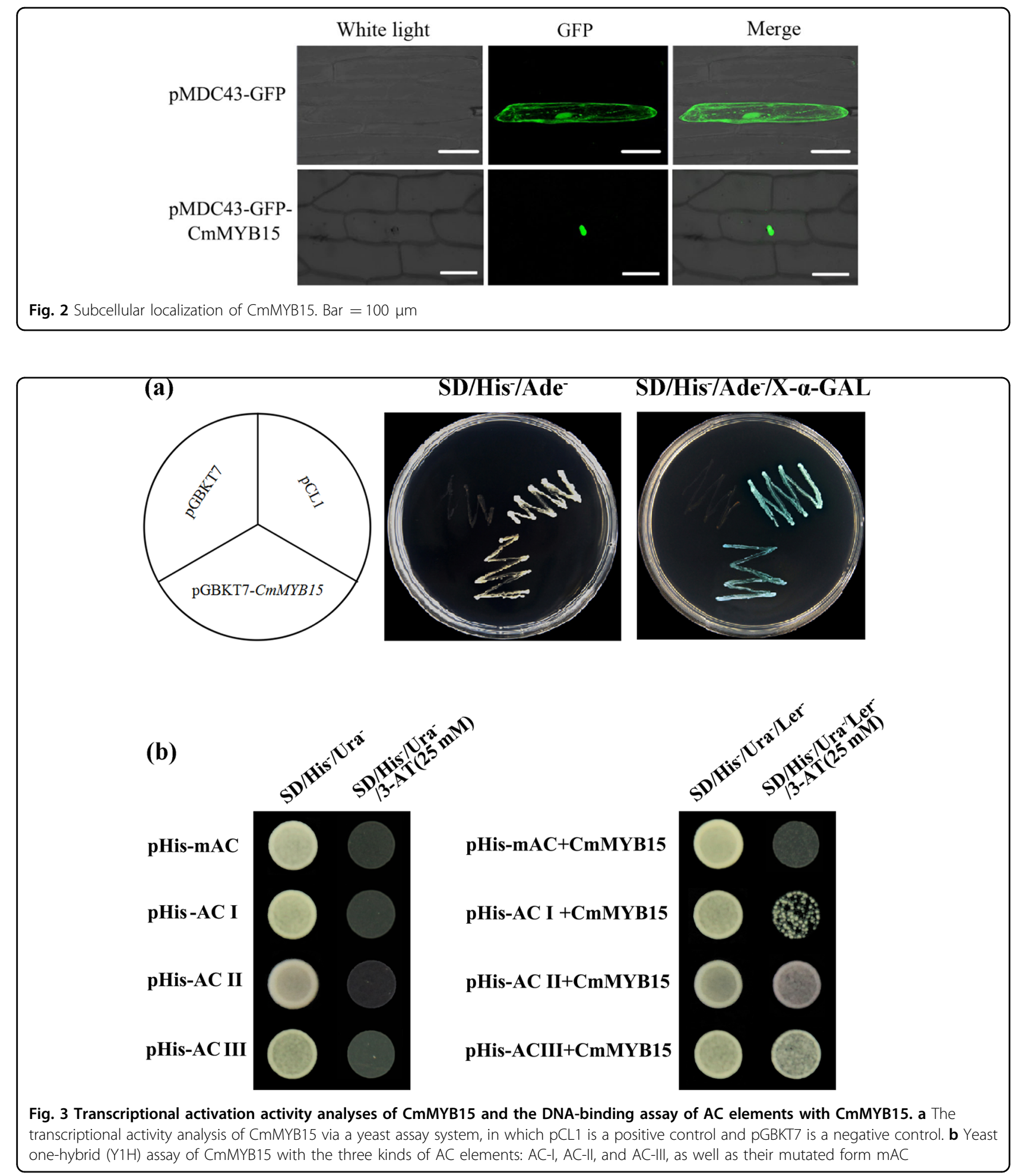

\section{Discussion}

\section{CmMYB15 is a MYB transcription factor}

MYB transcription factors, which are widely distributed in plants, play an important role in regulating a variety of processes in plants ${ }^{1}$. MYBs act as transcription activators or repressors to regulate the transcription of downstream genes. Of all the divisions of plant MYB proteins, including 4R-MYB, 3R-MYB, R2R3-MYB and MYBrelated types, R2R3-MYB proteins account for the majority ${ }^{24}$. Most R2R3-MYB and 3R-MYB subfamilies in 


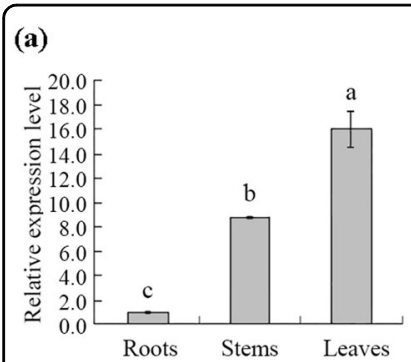

(b)

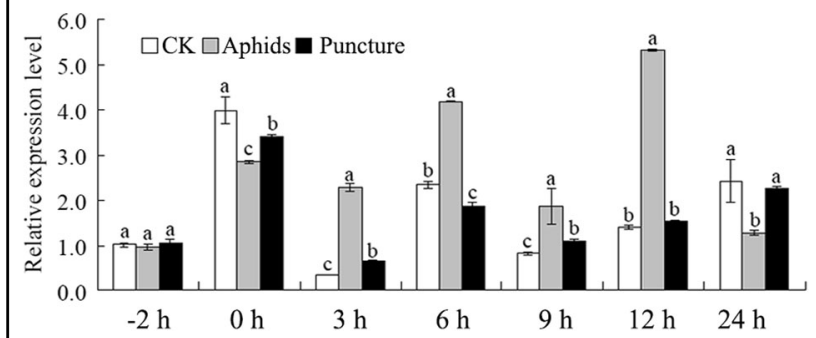

Fig. 4 Expression of CmMYB15 in different organs of chrysanthemum plants and under aphid and puncture treatment, as revealed by quantitative real-time PCR.

a Expression of CMMYB15 in the roots, stems and leaves of wild-type chrysanthemum, $\mathbf{b}$ Expression of CMMYB15 in response to aphid treatment and puncture treatment in the third fully expanded leaf counted from the apex. The bars indicate the standard errors

Arabidopsis are divided into 16 groups with different functions, such as involvement in stress responses, primary and secondary metabolism, lignin biosynthesis or wall deposition, cell wall thickening, cell differentiation or embryogenesis, the cell cycle, anther development, stomatal development and meristem formation ${ }^{3,25}$. Previously, only a few MYB transcription factors in chrysanthemum had been isolated and analyzed. Among them, chrysanthemum CmMYB1 is a repressor that reduces the lignin content and represses flavonoid synthesis in Arabidopsis ${ }^{26}$. Chrysanthemum CmMYB2 enhances the salinity and drought stress of Arabidopsis; additionally, the ABA sensitivity and flowering period of plants is affected by this $\mathrm{MYB}^{27}$. Chrysanthemum CmMYB6 was found to be involved in anthocyanin biosynthesis $^{28}$. In this study, a new MYB family gene in chrysanthemum, CmMYB15, was isolated. Sequence analysis showed that $C m M Y B 15$ contains two conserved MYB domains, which are typical of R2R3-MYB genes. Cluster analysis showed that $C m M Y B 15$ is closely related to AtMYB15, AtMYB13, AtMYB58, and AtMYB63. These genes are thought to belong to a group related to stress responses $^{29}$. In addition, CmMYB15 was found by GFP localization analysis to be expressed in the cell nucleus, which is expected given its role in transcriptional activation.

\section{Overexpression of CmMYB15 enhanced aphid resistance in chrysanthemum}

Plants are commonly exposed to many forms of abiotic and biotic stresses during growth. In response to these stresses, plants have evolved various defense mechanisms. MYBs are involved in the response to abiotic stress, including salt, drought, and high or low temperature ${ }^{30-32}$. For example, AtMYB20 can enhance salt tolerance ${ }^{33}$, while AtMYB73 suppresses salt overly sensitive (SOS) genes and is a negative regulator of salt responses ${ }^{34}$. Several MYBs, such as AtMYB74 and AtMYB88, participate in both salt and drought stress responses ${ }^{35-37}$. AtMYB15, a homologous gene of $C m M Y B 15$, enhances drought and salt tolerance by improving sensitivity to abscisic acid, but it weakened the freezing tolerance of Arabidopsis by suppressing the expression of $C B F 1 /$ $D R E B 1$, which activated cold-responsive genes ${ }^{38,39}$. On the other hand, the Fagopyrum tataricum MYB gene FtMYB15 enhances the synthesis of anthocyanins and proanthocyanidins, which help resist abiotic stress ${ }^{40}$. Similarly, SIMYB75 in tomato also contributes to the accumulation of anthocyanins and participates in several stresses responses ${ }^{41}$.

At present, the MYBs involved in the response to biotic stresses such as pathogen attack and pest feeding have received increased amounts of attention. By promoting the biosynthesis of salicylic acid, AtMYB96 induces pathogen resistance in Arabidopsis ${ }^{42}$. The role of MYBs in insect defense has been recognized; for example, AtMYB102 is expressed locally at the feeding sites of Pieris rapae in damaged leaves, which then activates the expression of defense-related genes and cell wall modification genes ${ }^{43}$. Overexpression of AtMYB12 in Nicotiana tabacum enhances the expression of phenylpropanoid pathway genes and the accumulation of flavonols, especially rutin, consequently leading to insect resistance $^{31}$. In the present study, CmMYB15 was significantly induced by aphid infestation. CmMYB15-overexpressing plants exhibited a lower average multiplication rate and higher average inhibition ratio of aphids, in which CmMYB15-ox3 lines, which presented a relatively high expression of the CmMYB15 gene, showed a higher IR than CmMYB15-ox4 lines, which presented relatively low CmMYB15 expression. These results indicated that CmMYB15 contributed to the resistance of chrysanthemum to aphids.

\section{CmMYB15 enhanced aphid resistance by increasing lignin synthesis}

When faced with phytophagous pests, plants can enhance their physical structures and alter their secondary metabolites to hinder the invasion of pests or inhibit pest growth and reproduction. Research in rice (Oryza 
(a)

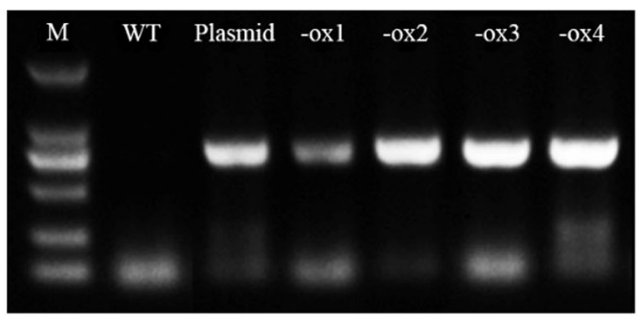

(b)

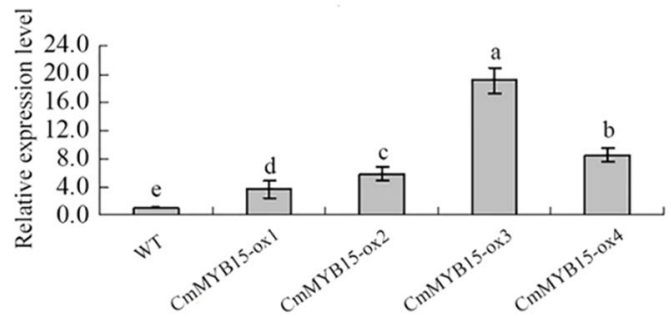

(c)
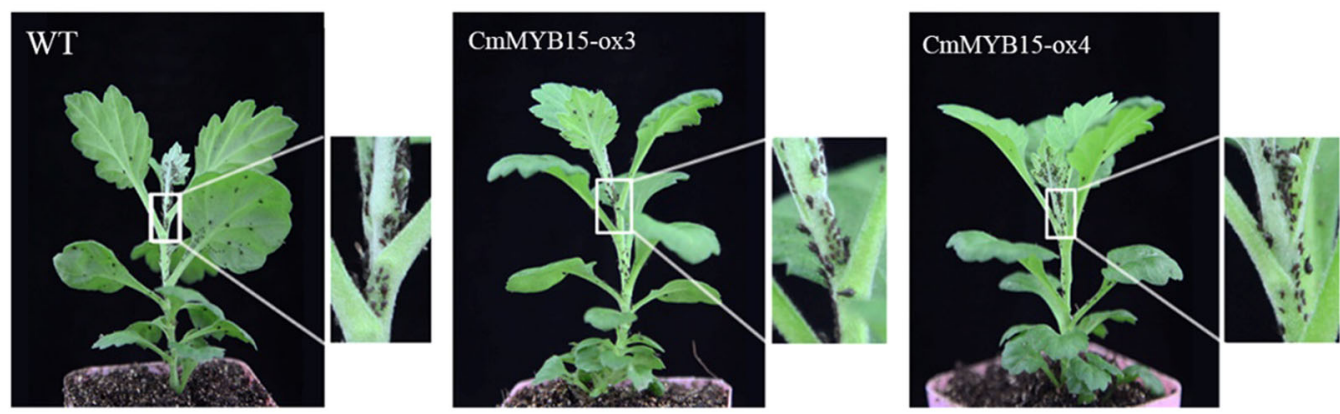

(d)

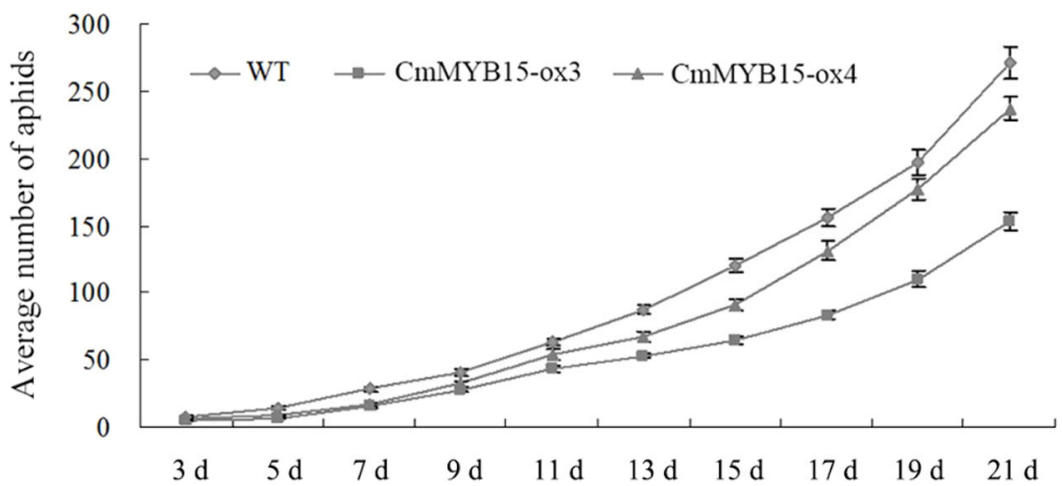

Fig. 5 The aphid resistance of wild-type chrysanthemum and CmMYB15-overexpressing chrysanthemum. a Identification of CmMYB15overexpressing chrysanthemum plants by PCR, in which the plasmid pMDC43-2 355 5::CmMYB15 acted as a positive control and wild-type (WT) acted as a negative control. -ox1, -ox2, -ox3 and -ox4 represent transgenic plants overexpressing CMMYB15, b transcription level of CmMYB15 in wildtype and CMMYB15-overexpressing chrysanthemum plants, $\mathbf{c}$ differential proliferation of aphids between CmMYB15-overexpressing and nontransgenic plants, $\mathbf{d}$ average number of aphids on CMMYB15-overexpressing and wild-type plants measured at 3-21 days after aphid infestation

sativa) has demonstrated that three MYB genes, MYB30, $M Y B 55$, and MYB110, which are induced by pathogen infestation, are involved in plant primary immunity (known as a microbe-associated molecular pattern) by specifically inducing monolignol pathway genes ${ }^{44}$. In Arabidopsis, recent research has demonstrated that AtMYB102 promotes ethylene (ET) biosynthesis by upregulating ET biosynthesis pathway genes and increases the susceptibility of plants to aphids ${ }^{45}$. Phloem-based defense (PBD) regulated by ethylene (ET) and MYB transcription factors is a mechanism that has been studied substantially in plants with specific regard to how plants are resistant to insects such as aphids. Sieve-specific phloem proteins (PP) can plug sieve pores and prevent aphids feeding on the phloem, which is a physical plant defense mechanism ${ }^{46}$. In wheat (Triticum aestivum), TaMYB19, TaMYB29, and TaMYB44 act as coregulators of PBD, which is activated by the infestation of English grain aphids via crosstalk with the ethylene signaling pathway ${ }^{46}$. Three Arabidopsis mutants, atmyb15, atmyb38, and atmyb44, are susceptible to aphid infestation due to the disruption of ethylene signaling ${ }^{47}$, with AtMYB44 playing a dominant role in activating EIN2affected defense, an ethylene signaling regulation defense mechanism, to control resistance to the green peach aphid Myzus persicae Sulzer. This defense requires ethylene- 
Table 1 Comparison of aphid populations on wild-type plants and transgenic chrysanthemum plants overexpressing CmMYB15 after infestation

\begin{tabular}{lll}
\hline Plants & $\begin{array}{l}\text { Multiplication rate (MR) } \\
\text { of the aphid population }\end{array}$ & $\begin{array}{l}\text { Inhibition rate (IR) of } \\
\text { the aphid population \% }\end{array}$ \\
\hline WT & $54.17 \pm 2.286^{\mathrm{a}}$ & 0 \\
CMMYB15-ox3 & $30.69 \pm 1.422^{\mathrm{c}}$ & 43.34 \\
CMMYB15-ox4 & $47.36 \pm 1.724^{\mathrm{b}}$ & 12.57 \\
\hline
\end{tabular}

Multiplication rate (MR) of the aphid population was calculated by $N_{21} / 5$, in which $N_{21}$ represents the average number of aphids on WT plants or transgenic plants on the 21 st day after aphid infestation. The inhibition rate (IR) of aphids on the transgenic plants was given by the formula $\left(N_{W}-N_{O}\right) / N_{W} \times 100$, in which $N_{W}$ and $N_{O}$ represents the number of aphids on the WT and on the CmMYB15overexpressing plants at 21 days after aphid infestation, respectively 22

The different letters $\left({ }^{\mathrm{a}}, \mathrm{b}, \mathrm{c}\right)$ represent significantly different aphid multiplications between the wild-type and CMMYB15-overexpressing plants

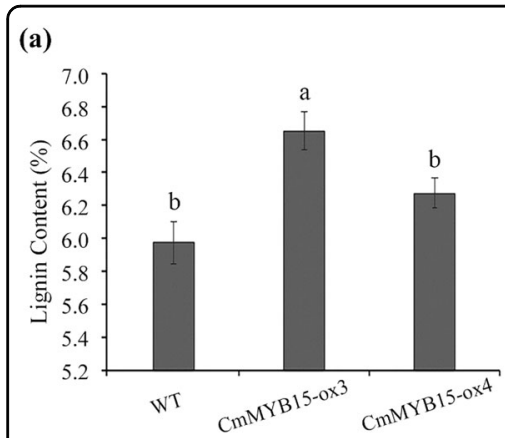

(b)

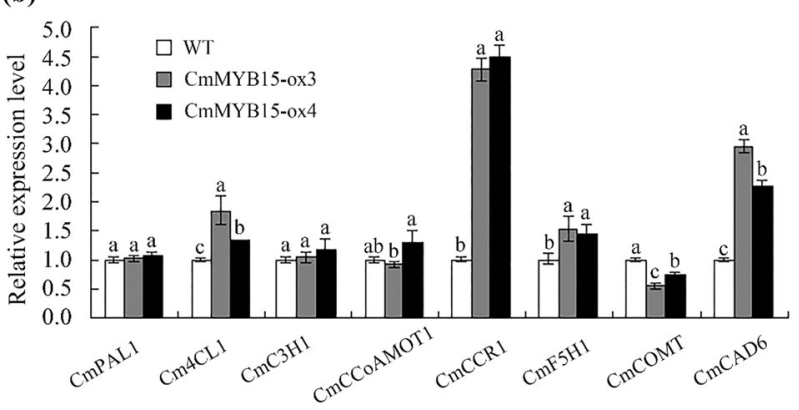

Fig. 6 The lignin content and expression levels of lignin biosynthesis genes in CmMYB15-overexpressing plants. a The lignin content of wild-type and CMMYB15-overexpressing plants, b expression levels of eight lignin biosynthesis genes in wild-type and CmMYB15-overexpressing plants. The bars indicate the standard errors

induced resistance involving the PBD response ${ }^{48-50}$. However, the mechanism of AtMYB15 in aphid resistance has not been further studied. Recently, it was determined that $A t M Y B 15$ regulates the biosynthesis of G-lignin to promote defense-induced lignification in Arabidopsis in response to pathogens, with AtMYB15 interfering with flg22, the bioactive epitope of bacterial flagellin ${ }^{6}$. Our previous study showed that $C m M Y B 19$, an aphid stress response gene, promoted lignin synthesis and resistance to aphids ${ }^{21}$. Here, we found that $C m M Y B 15$, a member of the MYB family, also enhanced aphid resistance and lignin synthesis. However, different sets of lignin genes were induced downstream in CmMYB19- and CmMYB15overexpressing plants. Cm4CL1 and CmCCR1 were induced in both $C m M Y B 19$ and $C m M Y B 15$ overexpression plants, while $C m F 5 H 1$ and $C m C A D 6$ were induced only in the $C m M Y B 15$ overexpression plants. CmPAL1, CmC4H, CmHCT and CmCCoAOMT1 were enhanced by overexpression of $C m M Y B 19^{21}$; however, the expression level of $C m M Y B 19$ was relatively little affected by $C m M Y B 15$ (Fig. S1), suggesting that the regulatory mechanisms of lignin biosynthesis of $C m M Y B 15$ and CmMYB19 were different from each other to some extent. We suppose that $C m M Y B 15$ might mainly regulate the synthesis of lignin monomers and that $C m M Y B 19$ might contribute to the very upstream steps of lignin biosynthesis and the assembly of lignin monomers; this speculation should be validated in our ongoing research.

Yeast assays showed that CmMYB15 was able to bind to the $\mathrm{AC}$ elements in promoter regions of some lignin pathway genes, suggesting a role for CmMYB15 in the activation of lignin pathway genes. However, whether the elevation of genes involved in lignin biosynthesis is directly or indirectly regulated by $C m M Y B 15$ is not currently known. In conclusion, increasing CmMYB15 expression could lead to changes in lignin content, thereby enhancing the aphid resistance of chrysanthemum, which provides much promise for improving physical defense to aphids.

\section{Materials and methods Plant materials}

'Nannong Xunzhang' (aphid resistant) and 'Jinba' (aphid susceptible) chrysanthemum plants were obtained from the Chrysanthemum Germplasm Resource Preserving Centre (Nanjing Agricultural University, China). The plants were grown under conditions of $25^{\circ} \mathrm{C}$ and $100 \mu \mathrm{mol} \mathrm{m}^{-2} \mathrm{~s}^{-1}$, with a $16 / 8 \mathrm{~h}$ light/dark photoperiod.

\section{Gene isolation and sequence analysis}

Total RNA from 'Nannong Xunzhang' chrysanthemum was isolated using RNAiso Plus Reagent (TaKaRa, Japan). First-strand cDNA was synthesized by M-MLV reverse transcriptase (TaKaRa, Japan). The cDNA fragments of CmMYB15 were amplified using Phusion High-Fidelity DNA Polymerase (ThermoFisher, USA) with genespecific primers (Supplementary Table S1) based on the transcriptome data of 'Nannong Xunzhang' ${ }^{20}$. 3'-RACE ( $3^{\prime}$-random amplification of cDNA ends) and 5'-RACE were then performed to obtain the full-length $\mathrm{CDNA}$ as described previously ${ }^{21}$. The ORF of CmMYB15 was 
amplified using the gene-specific primers CmMYB15-F/R (Supplementary Table S1).

The homologous sequences of CmMYB15 were retrieved and analyzed using online BLAST tools from the NCBI (National Center for Biotechnology Information, USA). The sequences of MYB proteins were aligned using DNAMAN and Muscle software. A phylogenetic tree of CmMYB15 with its homologs in Arabidopsis was constructed using MEGA 7.0 with the maximum likelihood method (bootstraps $=1000$ ).

\section{Subcellular localization of CmMYB15}

The ORF of CmMYB15 was ligated into the vector pENTR1A using Kpn I and Xho I restriction enzymes to generate a pENTR1A-CmMYB15 vector. A pMDC43$C m M Y B 15$ vector driven by the $2 \times 35 \mathrm{~S}$ promoter was then constructed via recombination of pENTR1ACmMYB15 with pMDC43-GFP using LR Clonase II enzyme mix (Invitrogen, USA). By particle bombardment (PDS-1000; Bio-Rad, USA), the plasmid DNA of pMDC43-CmMYB15 was transformed into onion epidermal cells ${ }^{51}$. The GFP signal of the transformed cells was detected by laser scanning confocal microscopy (LSCM, Leica, Germany) after an overnight dark incubation at $22^{\circ} \mathrm{C}$ on Murashige and Skoog (MS) medium.

Yeast one-hybrid assay for the transcriptional activation activity and binding of AC cis-elements by CmMYB15

To detect the transcriptional activity of CmMYB15, pGBKT7-CmMYB15 was constructed via recombination using pENTR1A-CmMYB15 as the donor vector and pGBKT7 as the destination vector with the aid of LR Clonase II enzyme mix (Invitrogen, USA). pGBKT7CmMYB15, pGBKT7 (an empty vector, as the negative control) and a positive control (pCL1) were transformed separately into $\mathrm{Y} 2 \mathrm{H}$ competent cells (Clontech, USA) containing the GAL4/UAS system. The transformed cells were cultured as described previously ${ }^{21}$.

To identify whether CmMYB15 could bind AC elements, a yeast one-hybrid assay was performed using the Matchmaker Gold Yeast One-Hybrid Library Screening System (Clontech, USA) according to the manufacturer's protocol. Three forms of AC elements, i.e., AC-I (ACCTACC), AC-II (ACCAACC) and AC-III (ACCTAAC), are available thus $\operatorname{far}^{52}$. Therefore, the fragments containing three tandem repeat $\mathrm{AC}$ elements, AC-I (5'-TCCACCTACCTCCACCTACCTCCACCTACCCC-3' $3^{\prime}, \quad$ AC-II (5'-TCCA $\overline{C C A A C C C C T C C A C-~}$ CAACCCCTCCACCAACCCC-3'), and AC-III $\overline{\left(5^{\prime}-\right.}$ TCCACСТAACTCCACCTAACTCCACCTAACCC-3'), respectively, and their mutated form, $\mathrm{mAC}\left(5^{\prime}\right.$ TCCAAATATTTCCAAATATTTCCAAATATTCC-3'), were assembled into a pHISi vector at the EcoR I and $X b a$ I restriction enzyme cutting sites. The plasmids were transformed into a $\mathrm{Y} 1 \mathrm{H}$ Gold line after linearization by an Apa I restriction enzyme. The binding assay was performed as described previously ${ }^{21}$.

\section{Gene expression analysis by quantitative real-time PCR (qRT-PCR)}

To analyze the expression profiles of $C m M Y B 15$ in different tissues, the roots, the fourth internode of the stem and the third fully expanded leaf counted from the apex were harvested from 'Nannong Xunzhang'.

To dissect the expression profiles of CmMYB15 in response to aphid infestation, aphid infestation was performed as described by Xia et al. ${ }^{20}$. The time point when aphids were infested was defined as $-2 \mathrm{~h}$; thus, $0 \mathrm{~h}$ refers to $2 \mathrm{~h}$ after infestation. To mimic piercing by an aphid, a puncture treatment was performed on the third leaf of another group of plants as described previously ${ }^{51}$. A group of untreated plants served as the control (CK). All the treated leaves were covered by transparent and ventilated petri dishes ${ }^{19}$. For RNA isolation, leaves from each treatment were harvested from three individual plants at $-2,0,3,6,9,12$, and $24 \mathrm{~h}$. The aphids were removed when the leaves were harvested. Each test included three biological replicates.

qRT-PCR was performed using SYBR Premix Ex Taq II (TaKaRa, Japan) according to the manufacturer's instructions. The gene Elongation Factor $1 \alpha$ (CmEF1 $\alpha$, KF305681) was used as the reference gene ${ }^{53}$. The qRTPCR data were analyzed using the $2^{-\Delta \Delta C T} \operatorname{method}^{54}$.

\section{Overexpression of CmMYB15 in 'Jinba' chrysanthemum}

To identify the function of CmMYB15, pMDC43-2 $\times$ 35S::CmMYB15 was transformed into Agrobacterium tumefaciens strain EHA105 using the freeze-thaw method. Information on the in vitro regeneration of 'Nannong Xunzhang' chrysanthemum is not available; thus, a CmMYB15-overexpressing 'Jinba' chrysanthemum was produced by Agrobacterium-mediated transformation as described previously ${ }^{55}$. Hygromycin-resistant shoots were rooted on MS medium containing hygromycin $B$, and the putative transgenic plants were identified by PCR using HptII primer pairs (Supplementary Table S1). The expression levels of $C m M Y B 15$ in the putative overexpression lines and wild-type (WT) plants were measured using the primer pair CmMYB15-qF/qR (Supplementary Table S1).

\section{The resistance of CmMYB15-overexpressing chrysanthemum plants to aphid}

Two independent overexpression lines were used for an aphid resistance assay according to the methods of $\mathrm{Hu}$ et al. ${ }^{56}$. Transgenic plants and WT 'Jinba' plants at the 6-8leaf stage were used for aphid resistance assays. The infestation of aphids was performed according to the method of 
Li et al. ${ }^{55}$. Each infestation assay contained ten plants with three biological replications. The number of aphids was counted every 2 days. The average multiplication rate (MR) and average IR of the aphid population were calculated based on the aphid data counted over 21 days $^{21}$.

\section{The determination of lignin content and expression levels of lignin biosynthesis genes in CmMYB15-overexpressing plants}

The fourth internode of the overexpressing lines and WT 'Jinba' plants at the 14-to-16-leaf stage was sampled, dried and ground. The lignin content was analyzed using the acetyl bromide procedure. The samples were prepared following the description by Foster et al. ${ }^{23}$ The absorbance at $280 \mathrm{~nm}$ was determined using a microplate reader. The lignin content was then determined using the formula $\% \mathrm{ABSL}=[\mathrm{abs} \times(2 \mathrm{ml} \times 100 \%)] /[(15.69 \times$ $0.539 \mathrm{~cm}) \times$ weight], where abs (absorbance) represents the average absorbance of 3 reads.

The fourth internode of the overexpressing lines and 'Jinba' plants at the 14-to-16-leaf stage was harvested. The expression of lignin biosynthesis genes, including CmPAL1, Cm4CL1, CmC3H1, CmCSE, CmCCoAMOT1, CmCCR1, CmF5H1, CmCOMT, and CmCAD6, was measured by qRT-PCR as mentioned above using the primers listed in Supplementary Table S1.

\section{Statistical analysis}

Tukey's multiple range test $(P<0.05)$ and one-way analysis of variance were performed to determine the significance of the data. SPSS v19.0 was used for all statistical analyses.

\section{Acknowledgements}

This work was supported by the National Natural Science Foundation of China (Grant No. 31672192), the Program for Key Research and Development, Jiangsu, China (Grant No. BE2017318), the National Key Research and Development Program of China (2018YFD1000402), and the Fundamental Research Funds for the Central Universities (KJQN201812).

\section{Author contributions}

C.A., L.S., and S.C. conceived and designed the experiments. C.A., L.S., and X.D. performed the experiments. C.A., L.S., Y.W., and Y.Z. analyzed the data. A.S., J.J., Z.G., W.F., and F.C. contributed the reagents/plant materials/analysis tools. C.A. and S.C. wrote the manuscript. All authors read and approved the manuscript.

\section{Conflict of interest}

The authors declare that they have no conflict of interest.

Supplementary Information accompanies this paper at (https://doi.org/ 10.1038/s41438-019-0166-y).

Received: 17 February 2019 Revised: 10 May 2019 Accepted: 17 May 2019 Published online: 11 July 2019

\section{References}

1. Ambawat, S., Sharma, P., Yadav, N. R. \& Yadav, R. C. MYB transcription factor genes as regulators for plant responses: an overview. Physiol. Mol. Biol. Plants 19, 307-321 (2013).

2. Baldoni, E., Genga, A. \& Cominelli, E. Plant MYB transcription factors: their role in drought response mechanisms. Int. J. Mol. Sci. 16, 15811-15851 (2015).

3. Dubos, C. et al. MYB transcription factors in Arabidopsis. Trends plant Sci. 15, 573-581 (2010)

4. Huang, W. et al. Isolation and molecular characterization of thirteen R2R3-MYB transcription factors from Epimedium sagittatum. Int. J. Mol. Sci. 14, 594-610 (2012).

5. Du, H., Feng, B.-R., Yang, S.-S., Huang, Y.-B. \& Tang, Y.-X. The R2R3-MYB transcription factor gene family in maize. PLOS ONE 7, e37463 (2012).

6. Chezem, W. R., Memon, A., Li, F.-S., Weng, J.-K. \& Clay, N. K. SG2-type R2R3-MYB transcription factor MYB15 controls defense-induced lignification and basal immunity in Arabidopsis. Plant Cell tpc.00954.02016 (2017).

7. Weng, K. et al. Transcriptome of Erysiphe necator-infected Vitis pseudoreticulata leaves provides insight into grapevine resistance to powdery mildew. Hortic. Res. 1, 14049 (2014).

8. Ossipov, V., Haukioja, E., Ossipova, S., Hanhimäki, S. \& Pihlaja, K. Phenolic and phenolic-related factors as determinants of suitability of mountain birch leaves to an herbivorous insect. Biochem. Syst. Ecol. 29, 223-240 (2001).

9. Bellincampi, D., Cervone, F. \& Lionetti, V. Plant cell wall dynamics and wallrelated susceptibility in plant-pathogen interactions. Front. Plant Sci. 5, 228 (2014).

10. Zhou, J., Lee, C., Zhong, R. \& Ye, Z.-H. MYB58 and MYB63 are transcriptional activators of the lignin biosynthetic pathway during secondary cell wall formation in Arabidopsis. Plant Cell 21, 248-266 (2009).

11. Vanholme, R. et al. Caffeoyl shikimate esterase (CSE) is an enzyme in the lignin biosynthetic pathway in Arabidopsis. Science 341, 1103-1106 (2013).

12. Raes, J., Rohde, A., Christensen, J. H., Van de Peer, Y. \& Boerjan, W. Genomewide characterization of the lignification toolbox in Arabidopsis. Plant Physiol. 133, 1051-1071 (2003).

13. Zhong, R., Richardson, E. A. \& Ye, Z.H. The MYB46 transcription factor is a direct target of SND1 and regulates secondary wall biosynthesis in Arabidopsis. Plant Cell 19, 2776-2792 (2007).

14. Tian, Q. et al. Functional characterization of the poplar R2R3-MYB transcription factor PtoMYB216 involved in the regulation of lignin biosynthesis during wood formation. PLOS ONE 8, e76369 (2013).

15. $\mathrm{Xu}, \mathrm{Q}$. et al. Activator-and repressor-type MYB transcription factors are involved in chilling injury induced flesh lignification in loquat via their interactions with the phenylpropanoid pathway. J. Exp. Bot. 65, 4349-4359 (2014).

16. Tang, $X$. et al. Poplar PdMYB221 is involved in the direct and indirect regulation of secondary wall biosynthesis during wood formation. Sci. Rep. $\mathbf{5}$ 12240 (2015)

17. Goggin, F. L. Plant-aphid interactions: molecular and ecological perspectives. Curr. Opin. Plant Biol. 10, 399-408 (2007).

18. He, J. et al. Chrysanthemum leaf epidermal surface morphology and antioxidant and defense enzyme activity in response to aphid infestation. J. Plant Physiol. 168, 687-693 (2011).

19. Xia, X. et al. MicroRNA expression profile during aphid feeding in chrysanthemum (Chrysanthemum morifolium). PLOS ONE 10, e0143720 (2015).

20. Xia, X. et al. Gene expression profiles responses to aphid feeding in chrysanthemum (Chrysanthemum morifolium). BMC Genom. 15, 1050 (2014).

21. Wang, $Y$. et al. CmMYB19 over-expression improves aphid tolerance in Chrysanthemum by promoting lignin synthesis. Int. J. Mol. Sci. 18, 619 (2017).

22. Deng, $Y$. et al. Production and characterisation of the intergeneric hybrids between Dendranthema morifolium and Artemisia vulgaris exhibiting enhanced resistance to chrysanthemum aphid (Macrosiphoniellasanbourni). Planta 231, 693-703 (2010).

23. Foster, C. E., Martin, T. M. \& Pauly, M. Comprehensive compositional analysis of plant cell walls (lignocellulosic biomass) part I: lignin. J. Visualized Exp. 37, e1745 (2010).

24. Wilkins, O., Nahal, H., Foong, J., Provart, N. J. \& Campbell, M. M. Expansion and diversification of the Populus R2R3-MYB family of transcription factors. Plant Physiol. 149, 981-993 (2009). 
25. Wang, S. et al. Genome-wide identification and characterization of the R2R3MYB transcription factor superfamily in eggplant (Solanum melongena L.). Agric. Gene 2, 38-52 (2016).

26. Zhu, L. et al. The heterologous expression of the chrysanthemum R2R3-MYB transcription factor CmMYB1 alters lignin composition and represses flavonoid synthesis in Arabidopsis thaliana. PLoS ONE 8, e65680 (2013).

27. Shan, $H$. et al. Heterologous expression of the chrysanthemum R2R3-MYB transcription factor CmMYB2 enhances drought and salinity tolerance, increases hypersensitivity to ABA and delays flowering in Arabidopsis thaliana. Mol. Biotechnol. 51, 160-173 (2012).

28. Liu, X.f. et al. The identification of a MYB transcription factor controlling anthocyanin biosynthesis regulation in Chrysanthemum flowers. Sci. Hortic 194, 278-285 (2015)

29. Chen, N. et al. Identification of 30 MYB transcription factor genes and analysis of their expression during abiotic stress in peanut (Arachis hypogaea L.). Gene 533, 332-345 (2014).

30. Roy, S. Function of MYB domain transcription factors in abiotic stress and epigenetic control of stress response in plant genome. Plant Signal. Behav. 11 e1117723 (2016)

31. Misra, P. et al. Modulation of transcriptome and metabolome of tobacco by Arabidopsis transcription factor, AtMYB12, leads to insect resistance. Plant Physiol. 152, 2258-2268 (2010).

32. Wei, X. et al. TaPIMP2, a pathogen-induced MYB protein in wheat, contributes to host resistance to common root rot caused by Bipolaris sorokiniana. Sci. Rep. 7, 1754 (2017).

33. Cui, M. H. et al. An Arabidopsis R2R3-MYB transcription factor, AtMYB20, negatively regulates type $2 \mathrm{C}$ serine/threonine protein phosphatases to enhance salt tolerance. Febs Lett. 587, 1773-1778 (2013).

34. Kim, J. H. et al. Loss of the R2R3 MYB, AtMyb73, causes hyper-induction of the SOS1 and SOS3 genes in response to high salinity in Arabidopsis. J. plant Physiol. 170, 1461-1465 (2013).

35. $\mathrm{Xu}, \mathrm{R}$. et al. Salt-induced transcription factor MYB74 is regulated by the RNAdirected DNA methylation pathway in Arabidopsis. J. Exp. Bot. 66, 5997-6008 (2015).

36. Xie, Z., Li, D., Wang, L., Sack, F. D. \& Grotewold, E. Role of the stomatal development regulators FLP/MYB88 in abiotic stress responses. Plant J. 64, 731-739 (2010).

37. Denekamp, M. \& Smeekens, S. C. Integration of wounding and osmotic stress signals determines the expression of the AtMYB102 transcription factor gene. Plant Physiol. 132, 1415-1423 (2003).

38. Ding, Z. et al. Transgenic expression of MYB15 confers enhanced sensitivity to abscisic acid and improved drought tolerance in Arabidopsis thaliana. J. Genet. Genom. 36, 17-29 (2009).

39. Agarwal, M. et al. A R2R3 type MYB transcription factor is involved in the cold regulation of CBF genes and in acquired freezing tolerance. J. Biol. Chem. 281, 37636-37645 (2006)
40. Luo, $X$. et al. An R2R3-MYB Transcription Factor FtMYB15 Involved in the Synthesis of Anthocyanin and Proanthocyanidins from Tartary Buckwheat. J. Plant Growth Regul. 37, 76-84 (2018).

41. Jian, W. et al. SIMYB75, an MYB-type transcription factor, promotes anthocyanin accumulation and enhances volatile aroma production in tomato fruits. Hortic. Res. 6, 22 (2019).

42. Seo, P. J. \& Park, C. M. MYB96-mediated abscisic acid signals induce pathogen resistance response by promoting salicylic acid biosynthesis in Arabidopsis. New Phytol. 186, 471-483 (2010).

43. De Vos, M. et al. The Arabidopsis thaliana transcription factor AtMYB102 functions in defense against the insect herbivore Pieris rapae. Plant Signal. Behav. 1, 305-311 (2006).

44. Kishi-Kaboshi, M., Seo, S., Takahashi, A. \& Hirochika, H. The MAMP-responsive MYB transcription factors MYB30, MYB55 and MYB110 activate the HCAA synthesis pathway and enhance immunity in rice. Plant Cell Physiol. 59, 903-915 (2018).

45. Zhu, L., Guo, J., Ma, Z., Wang, J. \& Zhou, C. Arabidopsis transcription factor MYB102 increases plant susceptibility to aphids by substantial activation of ethylene biosynthesis. Biomolecules 8, 39 (2018).

46. Zhai, Y. et al. Three MYB genes co-regulate the phloem-based defence against English grain aphid in wheat. J. Exp. Bot. 68, 4153-4169 (2017).

47. Liu, R. et al. Thirty-seven transcription factor genes differentially respond to a harpin protein and affect resistance to the green peach aphid in Arabidopsis. J. Biosci. 35, 435-450 (2010).

48. Lü, B. B. et al. At MYB 44 regulates resistance to the green peach aphid and diamondback moth by activating EIN2-affected defences in Arabidopsis. Plant Biol. 15, 841-850 (2013).

49. Liu, R. et al. Transcription factor AtMYB44 regulates induced expression of the ETHYLENE INSENSITIVE2 gene in Arabidopsis responding to a harpin protein. Mol. Plant-Microbe Interact. 24, 377-389 (2011).

50. Will, T. \& van Bel, A. J. Induction as well as suppression: How aphid saliva may exert opposite effects on plant defense. Plant Signal. Behav. 3, 427-430 (2008).

51. von Arnim, A. Subcellular localization of GUS-and GFP-tagged proteins in onion epidermal cells. Cold Spring Harb. Protoc. 2007, prot4689 (2007).

52. Ma, D. \& Constabel, C. P. MYB repressors as regulators of phenylpropanoid metabolism in plants. Trends Plant Sci. 24, 275-289 (2019).

53. Li, F. et al. Chrysanthemum Cm HSFA 4 gene positively regulates salt stress tolerance in transgenic chrysanthemum. Plant Biotechnol. J. 16, 1311-1321 (2018).

54. Livak, K. J. \& Schmittgen, T. D. Analysis of relative gene expression data using real-time quantitative $P C R$ and the $2^{-\Delta C T}$ method. Methods 25, 402-408 (2001).

55. Li, P. et al. The over-expression of a chrysanthemum WRKY transcription factor enhances aphid resistance. Plant Physiol. Biochem. 95, 26-34 (2015).

56. $\mathrm{Hu}, \mathrm{H}$. et al. Modification of chrysanthemum odour and taste with chrysanthemol synthase induces strong dual resistance against cotton aphids. Plant Biotechnol. J. 16, 1434-1445 (2018). 